livraisons

d'Histoire

de l'Architecture

\section{Livraisons de l'histoire de l'architecture}

$22 \mid 2011$

Émotions patrimoniales II

\title{
Entretien entre Daniel Fabre et Christian Hottin : le patrimoine saisi par l'événement
}

Heritage in the Sway of Events

Emotionen und Kulturerbe. Denkmalschutz als Zeitereignis, ein Gespräch

zwischen Daniel Fabre et Christian Hottin

Daniel Fabre et Christian Hottin

\section{(2) OpenEdition}

Journals

Édition électronique

URL : http://journals.openedition.org/lha/290

DOI : $10.4000 /$ /ha.290

ISSN : 1960-5994

Éditeur

Association Livraisons d'histoire de l'architecture - LHA

Édition imprimée

Date de publication : 10 décembre 2011

Pagination : $51-58$

ISSN : 1627-4970

Référence électronique

Daniel Fabre et Christian Hottin, «Entretien entre Daniel Fabre et Christian Hottin : le patrimoine saisi

par l'événement », Livraisons de l'histoire de l'architecture [En ligne], 22 | 2011, mis en ligne le 10

décembre 2013, consulté le 30 avril 2019. URL : http://journals.openedition.org//ha/290 ; DOI

$10.4000 /$ /ha. 290

Ce document a été généré automatiquement le 30 avril 2019.

Tous droits réservés à l'Association LHA 


\title{
Entretien entre Daniel Fabre et Christian Hottin : le patrimoine saisi par l'événement
}

\author{
Heritage in the Sway of Events \\ Emotionen und Kulturerbe. Denkmalschutz als Zeitereignis, ein Gespräch \\ zwischen Daniel Fabre et Christian Hottin
}

Daniel Fabre et Christian Hottin

Christian Hottin: Le thème de recherches des «émotions patrimoniales » intéresse le LAHIC depuis l'époque de la fondation du laboratoire. Pourriez-vous revenir sur les circonstances et les évènements qui ont conduit à la formulation de ce projet?

Daniel Fabre : En fait, l'attention à ce thème fut d'abord toute personnelle et elle est liée à un évènement bien précis dont je fus, par hasard et par chance, le témoin. En mai 1989 éclatait à Toulouse ce qu'il est convenu de nommer "l'affaire des mirandes ». À l'occasion de la restauration de Saint-Sernin, l'architecte en chef des monuments historiques qui en était chargé, Yves Boiret, conçoit comme une dérestauration de la restauration précédente celle que Viollet-le-Duc avait conduite en 1860. Après une bataille d'experts qui avait eu pour cadre la commission supérieure des monuments historiques, on aurait pu croire que la décision de dérestaurer allait s'appliquer sans problème. Or, comme vous le savez, dès que les travaux débutèrent, en mai 1989, les adversaires du projet mirent en œuvre tous les moyens pour contrecarrer leur avancée. Il se trouve que je dirigeais alors un laboratoire de recherche, le centre d'anthropologie, situé à deux pas de Saint-Sernin et dans le même bâtiment que la DRAC de MidiPyrénées. Plusieurs de mes collègues, enseignants à l'université du Mirail, avaient pris fait et cause pour Marcel Durliat, forte personnalité universitaire toulousaine, très opposé à la dérestauration; ils m'entretenaient de l'affaire que je voyais se développer tous les jours, sous mes yeux. 


\section{C.H. Quelles formes prenait-elle?}

D.F. Toutes les formes imaginables! Des débats d'experts dont La Dépêche du Midi donnait la chronique (au plus fort de l'affaire, qui dura une année, il y avait une page spéciale quasiment tous les jours); des manifestations de rue, en particulier autour de la basilique ; une pétition que l'on allait signer devant le parvis de l'église occupée. En fait, deux caractéristiques m'intriguaient considérablement. D’abord, l'apparente disproportion entre l'objet en cause - soit le remplacement des ouvertures situées sous le toit de la nef, donc vraiment dans les hauteurs peu visibles de l'édifice; Viollet-le-Duc leur avait donné la forme de losanges allongés, Boiret préférait une forme plus romane - et l'ampleur et l'intensité de la mobilisation. Ensuite, le processus d'élargissement progressif et de plus en plus rapide des acteurs engagés et des sphères impliquées. D'une part, le noyau militant, au départ plutôt académique et âgé, était enrichi de jeunes porteurs d'un style de vie et de création artistique alternatifs (certains de ces musiciens étaient par ailleurs mes étudiants en ethnologie), de l'autre, l'échelle du mouvement ne cessait de s'accroître - du local au national et même à l'international, du débat de spécialistes à l'engagement de n'importe quel citoyen, du patrimonial au politique...

C.H. Comment êtes-vous passé de l'étonnement à l'envie de comprendre?

D.F. Ce ne fut pas immédiat et il faudra pour cela un assez long détour. D’abord, à peine plus tard, je fus amené à suivre une autre affaire assez similaire, à propos de l'élargissement du parvis de l'église Saint-Vincent à Carcassonne. Je n'habitais plus cette ville mais ma mère, qui défila pour sauver le parvis, me gardait les journaux locaux et me faisait la chronique d'une lutte sans issue, là aussi. Par ailleurs, nous étions quelques-uns à proposer, au sein du conseil du patrimoine ethnologique, dont je fus nommé président en 1993, une réorientation partielle des intérêts du Conseil vers l'analyse anthropologique des pratiques patrimoniales. D'ailleurs, lorsque Gérard Ermisse devint le chef de la mission du patrimoine ethnologique et, à ce titre, s'occupa du colloque annuel ${ }^{1}$, nous avons suscité des débats et $\mathrm{y}$ avons pris part en essayant d'introduire, avec plus ou moins de succès, cette dimension. Je me rappelle, entre autres, du colloque de 1998, placé sous la présidence de Jacques Le Goff, et consacré aux passions patrimoniales ${ }^{2}$.

C.H. Le thème était donc dans l'air et l'intention partagée?

D.F. En fait, pas du tout, et c'est là le paradoxe. La brillante série de colloques annuels placés sous des présidences prestigieuses (celle de mes collègues de l'EHESS : Pierre Nora, François Furet, Jacques Le Goff, puis celle de Régis Debray etc.) avait pour but, dans l'esprit de la direction du Patrimoine qui en était le commanditaire, de réfléchir sur «l'inflation patrimoniale » ainsi que sur les moyens de la « refroidir » et d'alléger l'engagement de l'État. Maryvonne de Saint-Pulgent, qui était alors aux commandes, avait ce souci et le discours dominant allait dans ce sens : comment éviter la frénésie patrimoniale que l'État lui même avait consacrée ? Vous noterez que les contributions des ethnologues dans ces colloques publiés sont le plus souvent en porte-à-faux, puisque elles sont résolument descriptives et pas le moins du monde normatives. Il s'agissait de comprendre ce qui se passait dans les pratiques ordinaires du patrimoine et non de donner des avis sur la politique à suivre. 


\section{C.H. Mais alors, comment a émergé le thème des émotions comme terrain d'enquête?}

D.F. Par la bande, comme il arrive souvent. Une de mes doctorantes, Bérénice Waty, spécialiste de la lecture, souhaitait aborder un terrain très différent. Elle a été candidate a une bourse de la direction du Patrimoine et elle s'est lancée en 1999 dans une enquête rétrospective sur la dérestauration de Saint-Sernin. D'autre part, des collègues sociologues rennais avaient produit une étude immédiate sur l'incendie du Parlement de Bretagne a Rennes. L'évènement est de février 1994, leur livre est sorti en $1999^{3}$. Par ailleurs, un étudiant toulousain travaillait sur la commémoration ratée de la fondation de l'Université de Toulouse, en 1977... Des travaux donc, très peu nombreux en ces débuts, permettaient d'esquisser une première typologie de ces situations que le terme « émotions » permettait non seulement de regrouper commodément mais aussi de penser ensemble.

C.H. Pourquoi «émotions » et non pas «controverses», «affaires» ou d'autres synonymes?

D.F. Justement parce que c'est le terme le plus englobant. Il reprend l'acception un peu oubliée $\mathrm{du} \mathrm{XVIII}^{\mathrm{e}}$ siècle où le terme désigne un phénomène collectif, une mise en mouvement spontanée et inattendue, qui conteste l'ordre établi et exerce à son égard une certaine violence, directe ou symbolique. Je me suis à plusieurs reprises attaché à décliner les dérivations du terme et elles sont très riches. D'abord « émotion » est à la charnière du collectif et de l'individuel, ce qui conduit à poser la question même de l'engagement. Et puis « émotion » peut s'exprimer dans des motions, se pérenniser en un mouvement etc. Le champ sémantique qui s'ouvre est très nuancé, très riche... Aussi, au moment de la fondation du LAHIC, en 2000, nous avons fait des émotions patrimoniales un des grands chantiers du laboratoire et en janvier 2001 je proposai une toute première typologie devant des collègues archéologues à Sienne, en Italie ${ }^{4}$.

C.H. Ce programme a pris corps à travers une série de réalisations concrètes: fiches documentaires, programme de recherche de la mission du patrimoine ethnologique, séminaires et journées d'études, faisant intervenir un grand nombre de chercheurs qui n'étaient pas forcément associés au projet initial. Comment ces modifications d'échelle et ces changements de médium ont-ils contribué à faire évoluer la problématique de départ?

D.F. Outre la recherche de Bérénice Waty sur Saint-Sernin, les émotions patrimoniales ont fait l'objet du premier séminaire collectif du LAHIC, au cours des années 2001-2002. On peut dire que l'objet a pris forme dans ces débats très suivis par tous les membres fondateurs du laboratoire. À cette occasion, nous avons d'abord élargi considérablement la série des cas examinés y incluant, par exemple, des affaires comme celle du fichier juif ou des colonnes de Buren. Ensuite, l'ensemble s'est internationalisé : de la destruction des Bouddhas de Bamian à la difficile récupération des traces du ghetto de Varsovie. Simultanément, nous avons réfléchi aux modalités d'approche de ces situations et nous nous sommes tout simplement demandé : comment les décrire sans laisser passer l'essentiel ? Ce qui est toujours une bonne question. Ensuite, nous avons invité dans le séminaire des collègues sociologues qui avaient été aux prises avec des phénomènes semblables et avaient tentés d'en tirer des outils méthodologiques et théoriques. Or il se trouve qu'une bonne part des sociologues attachés à sortir du modèle de la sociologie critique, incarnée par Pierre Bourdieu, travaillaient à construire une sociologie de la critique dont les débats et controverses étaient le thème central ; il convenait de montrer que les sujets sociaux n'étaient pas mus par des forces qui s'imposaient à eux du dehors (et du dedans puisqu'elles étaient incorporées dans l'habitus) mais que leur capacité d'argumentation, de mobilisation et d'action étaient 
présentes, insérées dans des situations et actions largement autonomes. Aussi, dès 2001, avons-nous demandé à Nathalie Heinich (membre associé du laboratoire) qui avait étudié l'affaire des colonnes de Buren, à Philippe Urfalino, qui avait publié son ouvrage sur l'Opéra Bastille ${ }^{5}$, et à Francis Chateaureynaud qui mettait au point un logiciel de traitement des controverses de venir discuter avec nous de leurs analyses en s'en tenant au niveau de généralisation qui leur semblait possible, eu égard à leurs résultats. Cela donna lieu à des séances passionnantes. Bien entendu, il ne s'agissait pas pour nous de s'en tenir à une simple application de méthode ou à une simple vérification de la manière dont la société fonctionne; nous tenions à conserver à notre objet sa spécificité en montrant comment les «émotions patrimoniales» ne sont pas des affaires tout à fait comme les autres, puisque, justement, elles mettent en jeu les valeurs spécifiques que l'objet « patrimoine » cristallise aujourd'hui.

C.H. Mais qu'en est-il de l'aspect « inventaire appliqué » qui semble s'imposer asse vite?

D.F. Ce fut un moment important et qui mérite une réflexion approfondie; il est, en effet, toujours instructif de méditer les échecs. Dans le programme du tout nouveau LAHIC la mission du patrimoine ethnologique de l'époque avait repéré le possible intérêt immédiat de ce thème des émotions patrimoniales et avait convaincu la directrice de la DAPA, Wanda Diebolt, d'en faire un thème privilégié en créant même un observatoire de ces évènements. L'argument pour ce faire était astucieux: généralement l'administration ne voyait pas venir ces affaires, elle n'y tenait pas le beau rôle et les fonctionnaires, très compétents, qui les avaient à charge étaient toujours tiraillés entre l'application de règles et de décisions centrales et les spécificités de situations locales complexes, difficiles à saisir et tout a fait ignorées. Ce déficit grave incitait à un suivi plus attentif et plus régulier. De fait, indépendamment du LAHIC, Bérénice Waty fut chargée d'imaginer cet «Observatoire permanent des émotions patrimoniales » en mettant d'abord au point une fiche descriptive. Le résultat de son travail, consigné dans un rapport de décembre 2002, est fort intéressant puisqu'elle y souligne tous les obstacles que rencontre un pareil projet. Le principal étant que toutes les émotions, même lorsqu'elles naissaient de catastrophes mobilisatrices, débouchaient sur des controverses et des conflits dans lesquels l'ethnologue ne pouvait assumer la place du collecteur de renseignements destinés à alimenter une base de données au sein du ministère dont ils contestaient souvent les décisions. De plus, les acteurs semblaient tout disposés à interdire la publication de leurs documents. L'observatoire, voué à enregistrer des affaires closes, perdait beaucoup de son efficacité attendue. L'application administrative en venait à précéder la recherche et même, d'une certaine façon, à l'entraver. Soit le contraire du but, assez irénique, que l'on s'était donné au départ. En conséquence, il convenait d'arrêter les frais et de revenir à ce qui doit être toujours la première étape : conduire des recherches approfondies sur quelques cas bien choisis afin de se donner une connaissance réelle et actualisée du phénomène. C'est cette voie qui a ensuite été explorée puis poursuivie avec vous en lançant un programme de recherche qui a abouti a trois monographies solides : sur la tempête de 1999 dans le parc de Versailles, l'incendie du château de Lunéville et le projet de barrage dans la vallée des Camisards en Cévennes.

C.H. La question des émotions patrimoniales est une approche transversale de l'ethnologie du patrimoine, qui vient recouper les approches sectorielles développées dans d'autres projets et programmes du laboratoire. En quoi cette problématique peut elle apparaître 
comme une sorte de «fil rouge » structurant qui explicite l'ensemble du programme de l'équipe?

D.F. Le programme du LAHIC est entièrement contenu dans son intitulé : histoire et anthropologie de l'institution de la culture. Il repose sur un constat général qui voit dans l'évolution des sociétés modernes croître l'autonomisation de certains champs structurants de la vie sociale tel l'économique, le politique et le religieux, pour citer les trois domaines qui ont fait l'objet des enquêtes les plus décisives. Cette "grande transformation », pour reprendre l'expression de Karl Polanyi, touche également le domaine que l'on désigne aujourd'hui sous le nom commun de «culture ». Il n'est plus de société qui ne se revendique et ne se pense comme telle sans inclure au cœur même de sa définition un ensemble de pratiques, de sites, d'œuvres... grâce auxquels s'identifier personnellement et collectivement. La langue en fait partie, mais aussi la littérature, les arts, les paysages, le patrimoine dans ses divers contenus etc. Le mouvement même d'institution de la culture implique trois moments articulés mais distincts : la séparation de biens dont la qualification de "culturels » transforme le statut et, oserais-je dire, l'essence ; la médiation entre ces biens et leurs ayant-droit qui, idéalement et illusoirement, sont tous les membres d'une société ; l'identification de collectifs et d'individus par le truchement de ces biens sans maître que l'on s'approprie de multiples façons. L'échelle de ces processus a d'abord été nationale mais elle peut être aussi régionale, locale, professionnelle etc. Le fait nouveau est que cette production de biens particuliers, dits biens culturels, est aujourd'hui mondialisée et qu'il n'y a plus de société qui se veuille telle sans élection explicite de ce qui est, en son sein, considéré comme culture. Ce qui relativise les éternels débats sur ce qu'est la culture pour les anthropologues. Au cœur de ce processus se place un acte très difficile à appréhender, c'est l'attribution de valeur et donc l'introduction d'une qualification nouvelle ainsi que le bouleversement de l'échelle indigène des valorisations. On voit bien le rôle que les institutions étatiques ont joué dans ces qualifications et requalifications, dans leur définition, leur administration, leur gestion quotidienne, mais on mesure mal jusqu'à quel point et selon quelles modalités ces attributions de valeur ont été activement prise en charge par les "gens » auxquels elles semblent être imposées de haut. Les émotions patrimoniales nous conduisent donc au cœur du processus d'attribution de valeur et révèlent aussi bien ce qu'il y a de consensuel et ce qu'il peut y avoir de polémique dans ce geste qui élit, sépare et, plus ou moins, partage. Autrement dit, l'étude des émotions patrimoniales est un poste d'observation, central, décisif, du processus général d'institution de la culture. Mais il y a plus. L'énoncé des traits généraux du processus d'institution de la culture, tout en situant à leur juste place quantité de travaux particuliers sur le champ culturel, risque d'apparaître comme exagérément englobant. Non qu'il soit nécessaire de se rabattre nécessairement sur les micro-observations de cas : la perspective large me semble tout aussi indispensable, mais à condition que ses propositions les plus cruciales puissent être soumises à une approche empirique et faire l'objet d'enquêtes. Les émotions patrimoniales sont un excellent terrain pour deux branches de la sociologie récemment développées : celle qui s'intéresse aux affaires, débats et controverses en portant toute son attention sur les argumentations affrontées (ce qui réalise le programme descriptif contenu dans la très ancienne rhétorique), celle qui s'intéresse aux mobilisations et qui reprend à nouveaux frais les questions de la branche minoritaire de la sociologie française, du côté de Tarde et Le Bon pour simplifier. J'ajouterais, et c'est essentiel, qu'elles me semblent offrir, au-delà même des traits qui les rassemblent, un terrain exemplaire où mettre à l'épreuve toutes les 
grandes propositions qui constituent théoriquement le champ de l'institution de la culture. On peut les utiliser comme un des multiples exemples de la créativité stratégique des groupes mais leur véritable enjeu scientifique est, à mes yeux, d'éclairer le processus d'attribution de la valeur culturelle et donc de définition de celle-ci dans son ensemble. Et ce, en affirmant la puissance de l'ethnographie comme forme d'approche et source de problématisation.

C.H. Nombre de cas étudiés dans le programme de recherche renvoient aux années 1980-1990, décennies patrimoniales par excellence, de part et d'autre des commémorations de 1989. Peut-on penser que les émotions patrimoniales sont un objet historiquement daté, qui perdrait sa pertinence dans un contexte marqué par une moindre omniprésence de la notion de patrimoine dans le débat public?

D.F. La question est complexe, exigerait une recherche spécifique et mérite une réponse détaillée. D'abord, un mot sur le problème méthodologique qu'elle pose sans le souligner. L'étude des émotions prend place le plus souvent après que l'événement émotionnel a eu lieu, c'est-à-dire qu'elle en reconstitue l'émergence, l'intensité, le feu mais à distance, à partir des multiples traces que l'émotion a laissées, ainsi que de la mémoire des acteurs et des témoins pour qui l'enquête est une sorte d'anamnèse. D'où la recommandation d'observer, autant que possible, l'émotion en cours, ce qui oblige à se tenir à l'affût et à se rendre disponible. Ce n'est pas toujours faisable et cela nécessite une approche particulière. Pour ce qui est de l'historicité du phénomène, elle n'est pas contestable, on devrait pouvoir tracer une sorte de courbe des émotions qui figurerait leur intensité variable dans le temps... Mais attention aux effets d'optique. En fait, l'émotion, entendue comme mobilisation soudaine qui va toujours au delà du petit groupe des experts institutionnels, qui crée littéralement des groupes inédits ne vivant ensemble que dans la temporalité spécifique de la mobilisation, est consubstantielle à la notion de patrimoine et accompagne son émergence et son extension. Par exemple, le travail sur Saint-Sernin en 1990 a ramené au jour une émotion importante qui avait opposé, en 1860, le premier restaurateur de la basilique, l'illustre Viollet-le-Duc, et les membres de la société archéologique du Midi de la France, qui tenaient à conserver la prééminence sur le territoire où s'exerçait leur érudition. Sans doute y a $t$-il un changement d'échelle qui tient à la fois à la démocratisation du sentiment patrimonial (Aloïs Riegl la repérait bien en 1905, c'est un aspect essentiel de sa notion d'« ancienneté ») et à l'intense médiatisation de toute "affaire» locale puisque la presse vit de cela, cependant que l'on voit aujourd'hui Internet prendre le relais. En réalité le nombre des émotions, plus discrètes, qui ponctuent le passé est considérable et je suis prêt à parier qu'après la parution de nos travaux, les historiens vont en multiplier le nombre. Reste à comprendre la force de ce registre de l'émotionnel en matière de patrimoine. Elle tient au rapport de la notion, de la conscience et de l'action patrimoniales avec la situation de "perte ", c'est-à-dire d'amoindrissement de l'être et d'évidence irrémédiable de la mort. Il me semble que, loin de s'effacer, ce registre ne cesse de se réactiver et de s'étendre. Nous nous sentons tous aujourd'hui « concernés » par la disparition de la faune polaire et des paysages qui l'accueillaient, nous nous indignons de l'effacement annuel de dizaines de langues orales, nous n'admettons plus le décroît du nombre des espèces vivantes. Il y a dans cette rébellion une part de rationalité - ce sont les arguments écologiques sur la biodiversité - mais il y a surtout le pathétique de toute perte qui affecte chacun. Donc, contrairement à ce qu'un regard sur le temps court pourrait laisser entendre, l'émotion patrimoniale est en train de 
s'étendre et de se généraliser. D'où la puissance du thème et l'urgence d'en entreprendre l'exploration.

\section{NOTES}

1. Il s'agit des Entretiens du patrimoine.

2. Jacques Le Goff (dir.), Patrimoine et passions identitaires. Entretiens du patrimoines, Paris, Fayard et éditions du patrimoine, 1998, $445 \mathrm{p}$.

3. André Sauvage et Jean-Yves Dartiguenave, L'Incendie du Parlement de Bretagne : la genèse et l'écho d'une catastrophe, Rennes, éditions Apogée, 159 p.

4. Daniel Fabre, «Catastrofe, scoperta, intervento o il monumento come evento», Andreina Ricci (dir.), Archeologia e urbanistica, Florence, All'Insegna del Giglio, 2002, p.19-29.

5. Philippe Urfalino (éd.), Quatre voix pour un opéra : une histoire de l'Opéra Bastille racontée par Michèle, Audon, François Bloch-Lainé, Gérard Charlet, Michael Dittmann, Paris, A. M. Métailié, 1990, 310 p.

\section{RÉSUMÉS}

L'entretien apporte un éclairage sur le contexte intellectuel de la genèse du programme de recherche consacré aux émotions patrimoniales. Il donne également des indications sur la perception de ce phénomène par l'administration du patrimoine et sur le rôle joué par la mission du patrimoine ethnologique dans le projet d'observatoire des émotions patrimoniales. Enfin, il permet de mieux comprendre le rôle central de ce projet dans l'ensemble des axes de recherche du LAHIC. Dans l'exploration de l'anthropologie de la culture, la question de l'attribution de la valeur culturelle ou patrimoniale à un élément est essentielle : qui attribue cette valeur et comment est-elle attribuée? L'observation et la compréhension des mécanismes de tensions à l'œuvre dans les émotions patrimoniales est un mode privilégié d'approche de ces questions.

The interview throws some light on the intellectual context at the origins of the research programme devoted to 'heritage emotions'. It also gives some indications on the way this phenomenon is perceived by the heritage administration and on the role played by the mission for the ethnological heritage in the project for an observatory of heritage emotions. Finally, it helps understand the central role of this project in the broader lines of research undertaken by the LAHIC. In the exploration of the anthropology of culture, the question of how cultural or heritage value becomes attached to an element is essential. Who does this attributing and how exactly is the value attributed? The observation and understanding of the mechanisms of tension at work in heritage emotions is a particularly advantageous way of approaching these questions.

Durch dieses Gespräch wird ein besonderes Licht auf den intellektuellen Zusammenhang geworfen, der zur Entstehung eines Forschungsprogramms geführt hat, das sich der emotionalen 
Dimension des Kulturerbes (émotions patrimoniales) widmet . Dieses Gespräch bietet bedeutsame Erläuterungen einerseits über die Aufnahme dieses Phänomens durch die Verwaltung des Kulturerbes, andererseits über den besonderen Einsatz der Mission für das ethnologische Kulturerbe in das Projekt der Überwachung dieser emotionalen Dimension. Schließlich lässt es die zentrale Rolle dieses Projekts in den gesamten Richtlinien der Forschungen des LAHIC (Forschungslabor für Anthropologie und Geschichte der kulturellen Institutionen) besser erkennen. In der anthropologischen Forschung der Kultur geht es außerdem um die wesentliche Frage nach den Kriterien der Zuschreibung des Wertes an ein Kulturerbe. Die Kernfrage lautet : Von wem und wie wird dieser Wert verliehen? Die Beobachtung und das Verständnis für die internen Spannungen, die die émotions patrimoniales regieren, ermöglichen einen besonders günstigen Zugang zu diesen Fragen.

\section{AUTEURS}

\section{DANIEL FABRE}

Daniel Fabre est anthropologue, directeur d'études à l'École des hautes études en sciences sociales (Paris), depuis 1989 (chaire d'anthropologie de l'Europe). Il a été co-fondateur (avec Jean Guilaine, archéologue, professeur au Collège de France) du centre d'anthropologie des sociétés rurales, laboratoire de l'EHESS (1978) et du CNRS (1979), basé à Toulouse, et devenu en 1991 centre d'anthropologie de Toulouse, a dirigé ce centre jusqu'en 1997. Il est le fondateur et directeur du LAHIC (laboratoire d'anthropologie et d'histoire sur l'institution de la culture), actuellement équipe au sein de l'Institut interdisciplinaire d'anthropologie du contemporain. Il fut co-responsable de l'enseignement doctoral EHESS-École française de Rome (1995-2000) et professeur invité aux universités de Rome (La Sapienza, 1998-1999), professeur a contratto (Tor Vergata, 1999, 2000, 2001, 2002, visiting professor à l'Université di Roma 2-Tor Vergata (2003-2006). Dernier ouvrage paru : Les monuments sont habités (dir. avec Anna Iuso), Paris, éditions de la maison des sciences de l'Homme, 2010.

Christian Hottin est né en 1971. Il est archiviste paléographe, conservateur du patrimoine (Archives). Après avoir travaillé aux Archives nationales (Centre des archives du monde du travail de Roubaix), il a été chef de la mission ethnologie à la direction de l'architecture et du patrimoine de 2006 à 2010. Au sein de la direction générale des patrimoines, il est adjoint au département du pilotage de la recherche. Il participe aux recherches du LAHIC en tant que correspondant du ministère de la Culture.

Voir : http://www.iiac.cnrs.fr/lahic/spip.php?article339 